\title{
Proceso Semiquímico de Obtención de Pulpa de Eucalyptus camaldulensis; Dehn, Eucalyptus glóbulos: Labill y Eucalyptus viminalis; Labill, para Papel.
}

Semiquimic process for the obtaining of pulp of Eucalyptus camaldulensis; Dehn, Eucalyptus globulus; Labill and Eucalyptus viminalis; Labill for paper.

${ }^{1}$ Segundo H. Pérez Guardia; ${ }^{2}$ Segundo A. Cruz Hoyos

\section{RESUMEN}

El presente trabajo se realizó en la Universidad Nacional Agraria La Molina. Laboratorio de Pulpa y Papel, con muestras de Eucalyptus camaldulensis; Dehn, Eucalyptus glóbulus; Labill y Eucalyptus viminalis; Labill, procedentes del Valle del Mantaro, el objetivo fue determinar las características papeleras, utilizando el método semi-químico al sulfato. Las muestras se sometieron a una misma sulfidez de 11\%, al cabo de la digestión el desfibrado se realizó en las mismas condiciones de trabajo y metodología. El mayor rendimiento para Eucalyptus viminalis Labill (70.92\%) y el menor para Eucalyptus camaldulensis; Dehn (65.46\%), siendo la variación entre especies de $0.79 \%$, y una diferencia significativa entre especies. Las pulpas obtenidas se sometieron a $45^{\circ} \mathrm{SR}$ grados "shopper Riegler" de refinado, éste tratamiento a las fibras de pulpa, motivo incremento en longitud de rotura, índice de rasgado y doble plegado de Eucalyptus glóbulus, mayor deslignificación y blancura en pulpa, ocasionando menor gasto de reactivos para blanquear. Eucalyptus viminalis; Labill, Eucalyptus camaldulensis; Dehn en rendimiento en pulpa y composición química se encuentran dentro de los rangos apropiados para obtener pulpa semi-química al sulfato y papel para diferentes usos.

Palabras clave: Pulpa de Eucalyptus glóbulus, E. viminalis y E. camaldulensis.

\begin{abstract}
This work was carried out at the Universidad Nacional Agraria La Molina. Laboratory of Pulp and Paper, with samples of Eucalyptus camaldulensis; Dehn, Eucalyptus glóbulus; Labill and Eucalyptus viminalis; Labill, from the Mantaro Valley, the objective was to determine the pulp characteristics, using the semi-chemical sulfate method. The samples were subjected to the same sulfidity of $11 \%$, after the digestion the defibration was performed under the same working conditions and methodology. The highest yield for Eucalyptus viminalis Labill (70.92\%) and lowest for Eucalyptus camaldulensis; Dehn $(65.46 \%)$, being the variation between species of $0.79 \%$, and a significant difference between species. The obtained pulps were subjected to $45^{\circ}$ SR Riegler shopper grade, this treatment to the pulp fibers, reason increase in length of rupture, ripping rate and double folding of Eucalyptus glóbulus, greater delignification and whiteness in pulp, causing Less waste of bleaching reagents. Eucalyptus viminalis; Labill, Eucalyptus camaldulensis; Dehn in pulp yield and chemical composition are within the appropriate ranges to obtain semi-chemical sulphate pulp and paper for different uses.
\end{abstract}

Keywords: Pulp of Eucalyptus glóbulus, E. viminalis y E. camaldulensis.

'Universidad Nacional de Jaén. Jaén, Cajamarca, Perú

${ }^{2}$ Universidad Nacional de Jaén. Jaén, Cajamarca, Perú 


\section{INTRODUCCIÓN.}

Según la FAO, la demanda mundial de pulpa para papel registra un incremento constante y se prevé que los países productores tradicionales no podrán satisfacer en un momento dado y pronostican una grave escasez mundial de dichos productos. Sin duda sufrirán mayor escasez los países en vías de desarrollo, los cuales basan la mayor parte del consumo interno en importaciones. Frente a la creciente necesidad del producto papel a nivel mundial, las industrias enfocan los recursos madereros en las zonas tropicales, subtropicales y los bosques artificiales de frondosas es decir incursionando nuevas fuentes de materia prima para papel. El Perú debería ser autosuficiente en la producción de pulpa para papel, por cuanto tenemos grandes extensiones de plantaciones con la familia Myrtaceae, en la sierra y costa, destacando entre otras especies el eucalipto. Esta especie en los países de Sudáfrica, Brasil, Portugal y otros, se utiliza en la industria papelera. Sin embargo en nuestro medio la utilización de estas especies es de carácter primario.

En consecuencia con el presente trabajo de investigación, se pretende demostrar que estas especies serían las más apropiadas para cumplir con este objetivo, con buena calidad de madera fibrosa disponible a un precio relativamente bajo, su adaptación y su velocidad de crecimiento. El presente trabajo de investigación tiene como objetivos el estudio papelero de las especies Eucalyptus camaldulensis Dehn, Eucalyptus glóbulus Labill y Eucalyptus vaminalis Labil, con los siguientes objetivos específicos:

- Determinar las características químicas de pulpa, de Eucalyptus viminalis, Eucalyptus glóbulus; Labill y Eucalyptus camaldulensis; Dehn.

- Determinar el rendimiento de pulpa para papel, de las tres especies de eucalipto.

- Determinar el grado de refinado ajustado a 45

${ }^{\circ}$ SR para ensayos físico-mecánicos de pulpa.
La celulosa, hemicelulosa, la lignina, son polímeros que se encuentran en mayor y menor abundancia: tanino, materias colorantes, resinas aceites esenciales, oleorresinas, ceras, grasas, azucares, nitrógeno, minerales ácidos orgánicos libres, etc. La separación de estos componentes del tejido de la madera, presentan ciertas dificultades; es decir para disociar estos componentes orgánicos e inorgánicos es necesario recurrir a reactivos energéticos, los que tendrán una acción sobre los mismos cuerpos, necesitándose, además recurrir a pequeñas diferencias en las propiedades para separarlos (Navarro, 2006). Los componentes extraños de la madera, llamados extractivos que incluyen hidrocarburos alifáticos y aromáticos y sus derivados alcoholes; algunas maderas contienen aceites esenciales y aceites resínicos y esteroles; otras maderas poseen pequeñas cantidades de monosacáridos (Libby, 2000). El contenido de sustancias extraíbles varías según la edad y la duración del secado de la madera al medio ambiente. La finura de trituración es sumamente importante ya que tratándose de cuerpos con peso molecular elevado presentan dificultades al difundirse a través de las paredes celulares (Navarro, 2006). Por su parte (Libby, 2000). Afirma que los extractivos no se distribuyen uniformemente a lo largo del tronco del árbol, ni en una astilla individual.

El agua caliente actúa sobre los glúcidos, quedando en libertad radicales acetilos, en cambio los disolventes orgánicos separan del aserrín de la madera resinas, aceites, volátiles, taninos. Los solventes orgánicos disuelven la fracción de lignina. Con alcohol son extraídas ceras y grasas; presenta cierto inconveniente en disolver los azúcares y taninos. La extracción con benceno y disolventes clorados dan buenos resultados, pero no apropiados para materiales húmedos (Libby, 2000). (Goitia et al., 2005). En el interior de las células de la madera se encuentran una variedad de extractivos de 1-10 $\%$. 
Químicamente la celulosa es un carbohidrato, relacionado con los azúcares denominados polisacáridos, debido que su molécula contiene muchas unidades de glucosa que integra las cadenas de la celulosa. Bueno, 2001, afirma que las, maderas con alto contenido de celulosa, hemicelulosa y bajo contenido de lignina, extractivos, cenizas y sílice son óptimas para la obtención de pulpa para papel, que aquellas maderas de composición inversa. KIRT, 2001, señala que la lignina después de la celulosa es el principal componente de la madera, siempre asociado a la celulosa, carbohidratos $\mathrm{u}$ otros componentes; cuando se separan de otras sustancias la lignina es una sustancia polímero que tiene grupos hidroxilos y metoxilos, de cadenas ramificadas que pueden sustituir una red casi infinita. La celulosa se localiza principalmente en la zona de la lamela media, que funciona como relleno o sustancia cementante para impartir rigidez al tejido leñoso. Del total de los componentes de la madera, la lignina participa con $28 \%$ en las coníferas y $24 \%$ en las frondosas. Navarro, 2006, define a la lignina como el residuo no sacrificable desprovisto de productos accesorios como ceras, resinas, etc. También considera como la fracción de los tejidos lignificados.

La composición química de la madera del Eucalyptus glóbulus fue realizada por CARRUÑO \& VILLEGAS 2003, con fines papeleros, observaron su influencia de los componentes en las características del papel. El furfural proviene de pentosanos propiamente dichos, xilanos y arabanos de ácidos urónicos que acompañan a los polisacáridos, que son particularmente abundantes en la materia pépticas de la celulosa misma (Bueno, 2001). Los métodos utilizados en la práctica consiste en medir la cantidad de furfural producido por ataque de los pentosanos mediante ácido clorhídrico; lo pentosanos se hidrolizan en pentosas (xilanos y arabanos), estas a su vez sufren una descomposición (deshidratación), por acción el ácido, dando finalmente furfural (Navarro, 2006).

Las cenizas estas constituidas por residuos minerales obteniéndose por incineración de la materia prima (RODRIGUEZ et al., 2001). La ceniza es material mineral contenido en la madera, de donde podemos obtener en su totalidad o en parte de cada uno de los elementos existentes (Navarro, 2006).

La solubilización de la sílice hidratada en ácido es poco probable, pero en forma coloidal se puede filtrar a través del papel filtro, para el que se deshidrata, calcina y el residuo es la sílice (Bueno et al., 2001). La composición química de la madera de Eucalyptus glóbulus, procedente de Cajamarca, Cuzco y Junín es moderadamente variable, apta para ser utilizada en la fabricación de papel (Trujillo, 2001).

La pulpa semi-química o pulpa kraft al sulfato, es una subdivisión del proceso al sulfato, donde las astillas son semi-cocinadas, con la finalidad de producir fibras fuertes (GOITIA. 2005), la pulpa semi-química al sulfato es suficientemente ablandada, utilizando una solución de $\mathrm{NaOH}$ y $\mathrm{Na}_{2} \mathrm{~S}$ diluida, que permite una separación mecánica de las fibras, con rendimiento de 7085\% (KIRT, 2001). El nombre de pulpa Kraft, significa fuerte; obteniéndose papeles que sobrepasan en resistencia a cualquier papel; debido a la cocción incompleta de las astillas de la madera; siendo un tratamiento químicomecánico. Proceso que actualmente es utilizado industrialmente cada vez mayor, con maderas frondosas, con rendimiento de $70-85 \%$ en pulpa. Las condiciones utilizadas para la digestión de pulpa semi-química al sulfato es el siguiente: Reactivos $\left(\mathrm{Na}_{2} \mathrm{~S}+\mathrm{NaOH}\right)$, Álcali activo (9\%), Hidróxido de sodio (8\%), Sulfuro de sodio (1 $\%)$, Sulfidez (11\%), Reactivo deslignificación / madera seca (4.1), Temperatura de deslignificación $\left(160{ }^{\circ} \mathrm{C}\right)$, Periodo elevación de temperatura $\left(60^{\prime}\right)$, Periodo de deslignificación $\left(20^{\prime}\right)$, Cantidad de materia seca (300 gr. ), Tratamiento concluido (Desfibrado). 
Los porcentajes de reactivos son expresados como óxido de sodio $\left(\mathrm{Na}_{2} \mathrm{O}\right)$ (Libby, 2000), además los constituyentes básicos de la madera son: la celulosa, hemicelulosa, lignina y extractivos; que se eliminan en mayor y menor grado en los diversos procesos de tipo semiquímico, siendo el objetivo principal crear un debilitamiento químico del complejo ligninacarbohidratos en la unión entre fibras (tratamiento químico), parcialmente convertido en pulpa. El efecto de las variables en los procesos semi-químicos alcalinos influye directamente en el rendimiento y calidad de la pulpa. Los procesos semi-químicos, por lo general se efectúan con menor aplicación de álcali, con una concentración baja de reactivo y tiempo más corto (Libby, 2000).

Las variables que influyen en la segunda etapa del proceso semi-químico son: Material fibroso parcialmente convertido en pulpa, Contenido de humedad de material fibroso, Tamaño de astillas o de partículas, Temperatura en la zona de desfibrado Consistencia, Claro entre las placas (molino de disco de fricción), Número de pasos y Velocidad de alimentación o producción diaria (Bueno etal., 2001).

El refinado es una operación fundamental en la fabricación del papel, las fibras de la pulpa son sometidas a un tratamiento mecánico de golpeo, molido y fricción en presencia de agua, con cuya acción las fibras sufren diversos cambios morfológicos como rompimiento y demolición de la pared primaria, deformación y aplastamiento, fibrilación y acortamiento de las fibras, acompañado de cambios físico-químicos como: inhibición, hinchamiento e hidratación de las fibras, la que en estas condiciones adquieren mejores posibilidades de unión interfibrilar al exponer una mayor superficie al contacto con el agua (Costa, 2001) y (Bueno, 2001). Siendo fuertemente polar hacen que los hidroxilos de las cadenas celulósicas se orientan creando, en el momento de secado del papel, fuerzas de tal magnitud las cuales producen enlaces intermoleculares del tipo de puente de hidrógeno y fuerzas de Vander Waals, constituyendo la base de la cohesión y resistencia de la hoja formada. Por consiguiente estas hojas formadas adquieren un conjunto de propiedades físico-mecánicas, que las hacen aptas para convertirse en papeles de buena calidad; resaltando la resistencia a la tensión, dobles pliegue y al reventamiento (Fairest, 2004) y (De Los Heros, 2003).

El refinado influye directamente en las propiedades físico-mecánicas del papel; se manifiesta incrementando la resistencia a la tensión, dobles pliegues, estallido, transparencia y lisura del papel; mientras que la opacidad, blancura, porosidad, absorbencia y cuerpo decrecen (Pizarro, 2004).

La evaluación de sus características papeleras de la madera, de una especie forestal o de cualquier otro material lignocelulósico, se realiza mediante análisis químico, análisis biométrico de sus fibras y sus procesamientos para transformarla en papel, bajo una metodología establecida o con variantes (De Los Heros et al., 2003). La importancia tradicional de la longitud de las fibras en la fabricación del papel ha sido atribuida únicamente al entrelazamiento y filtrado natural de las fibras. Sin embargo últimos estudios demuestran, que la cohesión de la hoja de papel se hace básicamente a las atracciones de naturaleza electroquímica, desarrolladas a nivel molecular de las fibras, con enlaces de tipo hidrógeno y fuerzas de Vander Waals, son los que dan solidez al papel (Hinostroza, 2001) y (Molina, 2002).

El rendimiento, índice de deslignificación, blancura, opacidad, resistencia a la tensión, resistencia al rasgado y doble plegado, son parámetros principales que permiten emitir un juicio acertado sobre la aptitud papelera de una especie (Fairest, 2004).

Los análisis químicos de la pulpa, son índices que expresan el grado de deslignificación de una pulpa, también se puede determinar el contenido de lignina restante, utilizando reactivos 
oxidantes como: cloro hipoclorito y permanganato de potasio. Esta determinación se utiliza para pulpas sin blanquear, además permite verificar el proceso de cocción y lejiado de las materias celulósicas (Navarro et al., 2006). La determinación química en pulpa, tienen doble objetivo. El primero indica el grado de cocción de la pulpa. El segundo permite evaluar en consumo de licor o agentes blanqueadores. La composición de la lignina en la pulpa varía sensiblemente en comparación a su contenido original. Mientras menos drástica es la cocción, mayor es el blanqueo requerido (Grant, 2001).

Determinar el grado de cocción, consiste en oxidar la lignina contenida en la pulpa química o semi-química, el resultado depende del agente oxidante y la concentración del mismo. En el método de cloro, el agente oxidante es el cloro en forma gaseosa, agua de cloro o de hipoclorito de sodio y método de permanganato, se diferencia por la concentración de la solución del permanganato de potasio empleado, temperatura y por el tiempo de reacción, es el método más utilizado hasta ahora, por la sencillez y la rapidez de su ejecución (Torres, 2001).

(Trujillo, 2001), encontró en pulpa química al sulfato, procedente de (Cajamarca, Cusco y Junin), rendimientos de 51, 53,30 y $53 \%$ y bajo índice de cloro en muestras de Eucalyptus glóbulus de distintas procedencias, Cajamarca $51 \%$ de rendimiento, Cuzco (53 \%) y Junín (53 \%), con índice de Cloro $(1.53,1.28$ y 1.38$) \mathrm{y}$ índice de kappa $(11.42,10.10$ y 10.90) e Lignina residual $(1.48,0.48$ y 0.66$)$, significa que no tendrá dificultades en el blanqueo y que la demanda del blanqueador para esta operación será moderado.

\section{MATERIALES Y MÉTODOS.}

\section{Lugar de ejecución}

El presente trabajo se realizó en el Laboratorio de Pulpa y papel del Departamento Académico de Industrias Forestales de la Universidad Nacional Agraria La Molina.

\section{Materiales y equipos}

Los materiales fibrosos estudiados están constituidos por muestras de maderas de tres especies forestales: Eucalyptus camaldulensis Dehn, Eucalyptus glóbulos Labill y Eucalyptus viminalis Labill. Dichos materiales provienen de las plantaciones de eucalipto del Valle del Mantaro, departamento de Junín. La identificación de especies estuvo a cargo de la Sección de Dendrología del Departamento de Manejo Forestal, de la Universidad Nacional Agraria La Molina.

\section{Métodos y procedimientos}

Los métodos y procedimientos que se utilizaron para la obtención y evaluación de pulpa, semiquímica al sulfato.

\section{Preparación de muestras (astillas)}

Las muestras de madera han sido tomadas de 10 árboles por especie. De cada árbol se tomaron rodajas de 3 centímetros de largo; las que posteriormente se astillaron con las siguientes dimensiones: 3 centímetros de largo, $1-1.5 \mathrm{~cm}$ de ancho, $0.3-0.5 \mathrm{~cm}$ de espesor, de albura $\mathrm{y}$ duramen.

De cada troza de (E. glóbulus) astillada se tomaron $500 \mathrm{~g}$, posteriormente se realizó la mezcla, tratando de homogenizarlas, obteniéndose 15 kilos de astillas. E1 procedimiento fue igual para dos especies restantes.

\section{Obtención de pulpa}

Previa a la digestión, las astillas fueron remojadas en agua de caño durante 24 horas, dicho periodo permitió determinar la absorción de agua por las astillas.

Para obtener pulpa semi-química al sulfato, comprende de dos etapas. La primera es la parte química, con un tratamiento menos energético. La segunda corresponde a un tratamiento mecánico adicional de desfibrado. Las condiciones de digestión fueron los siguientes: Reactivos $\left(\mathrm{Na}_{2} \mathrm{~S}+\mathrm{NaOH}\right)$, Álcali activo (9\%), Hidróxido de sodio (8\%), Sulfuro de sodio (1 $\%)$ Sulfidez (11 \%), Reactivo de 
deslignificación/madera seca (4.1), Temperatura de deslignificación $\left(160{ }^{\circ} \mathrm{C}\right)$, Periodo de elevación de temperatura (60 min). Periodo de deslignificación (20min.), Cantidad de materia seca $(300 \mathrm{~g})$, Tratamiento concluido (Desfibrado)

Todos los reactivos empleados fueron expresados como oxido de sodio $\left(\mathrm{Na}_{2} \mathrm{O}\right)$. En seguida el cargado de los obuses al digestor, luego se puso en marcha el digestor, controlándose la presión y temperatura cada 10 minutos, durante el tiempo programado, de acuerdo el plan de trabajo.

\section{Lavado de la pulpa y cálculo de rendimiento}

Concluida la digestión, las astillas semi cocidas fueron descargadas de los obuses, para lavarlas con agua deshionizada sobre malla de acero inoxidable 150. Las astillas fueron desfibradas, hasta obtener pulpa. La pulpa obtenida pasó por una succionadora de agua y posteriormente son llevados al cripto perles para su homogenizado y pierda un poco de humedad. Para el cálculo de rendimiento de las pulpas obtenidas, se pesa la pulpa total y para determinar el porcentaje de humedad se pesan en cápsulas de porcelana tarada por duplicado, luego se llevan a una estufa de $105 \pm 2{ }^{\circ} \mathrm{C}$, hasta peso constante.

\section{Refinado}

El refinado de la pulpa se realizó en un refinador P.F.I, de acuerdo a la Norma SCAN -C: 18, a diferentes revoluciones, con la finalidad de encontrar 45 SR.

\section{Control de refinado}

Se realizó conforme a la Norma SCAN-M3: 65, mediante el método Shopper Riegler ( ${ }^{\circ} \mathrm{SR}$ ). Ajustadas a $45{ }^{\circ} \mathrm{SR}$, las pulpas obtenidas de las tres especies de eucalitpo

\section{Formación de hojas de ensayo.}

Después del secado al aire libre, las hojas se acondicionaron en un ambiente de humedad y temperatura controlada durante 24 horas $\left(20^{\circ} \mathrm{C}\right.$ y humedad relativa según Norma ITINTEC 272: 010).

\section{Propiedades físicos-mecánicas de la pulpa.}

Para el ensayo físico- mecánico de las hojas hechas en la formadora de laboratorio, se emplearon las siguientes normas de acuerdo a cada una de las determinaciones específicas:

Norma TAPPI 220 os - 71, G, Norma TAPPI 220 os - 71, E, Norma TAPPI 220 os - 71, P, Norma ITINTEC 272: 018, R, Norma TAPPI 220 os 71, Rp, Norma ITINTEC 272:027 Rr, Norma TAPPI 220 os - 71, Rs, Norma TAPPI 220 os 71, Db, Norma ITINTEC 272: 033.

\section{Análisis químico en pulpa}

Los análisis químicos en pulpa, se ejecutaron de acuerdo a los métodos especificados para cada caso:

Determinación del índice de cloro Norma SCAN C 29:72, Determinación de lignina residual método Jaime y chemp.

\section{Preparación de muestras de madera}

El análisis químico de madera de las tres especies. E. glóbulus Labil (9 trozas), E, viminalis Labill (9 trozas) y E. camaldulensis Dehn (9 trozas). Estas trozas se seccionaron (aserrío) por separado, durante este proceso se tomó 20 gramos de aserrín de cada troza. En total 180 gramos, que se homogenizaron adecuadamente, de acuerdo a la Norma TAPPI T220. Posteriormente se tamizó con malla $\mathrm{N}^{\mathrm{0}} 40 \mathrm{y}$ 60, utilizando para análisis químicos, la fracción que pasa por la malla $\mathrm{N}^{\circ} 40$ y las retenidas en la malla $\mathrm{N}^{\mathrm{o}}$ 60, de acuerdo a la TAPPI T6:11 m-59. Los resultados de cada ensayo se analizarán en diseño de bloques al azar y prueba de Duncan.

\section{RESULTADOS Y DISCUSIÓN}

En la tabla 1. Se presenta el ANVA para las variables evaluadas: 
Tabla 1. ANALISIS DE VARIANZA PARA LAS VARIABLES OBSERVADAS.

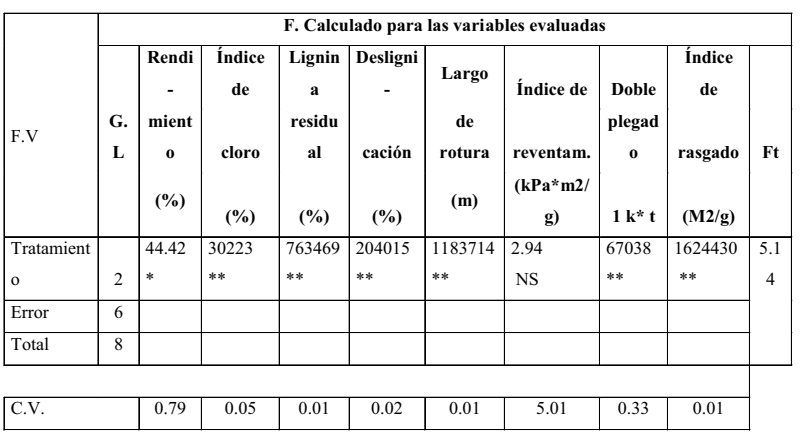

Se puede observar que en su fuente de variación tratamiento existe diferencia estadística significativa para todas las variables en estudio a excepción de índice de reventamiento, asimismo los valores del coeficiente de variabilidad están dentro del límite aceptable para un DCA.

En la tabla 2. Se presenta la prueba de comparación de medias de Duncan(0.05).

Tabla 2. Prueba de Comparacion de Medias para las Variables Observadas.

\begin{tabular}{r|c|c|c|c|c|c|c|c|} 
& & $\begin{array}{c}\text { Índice } \\
\text { de }\end{array}$ & Lignina & Desligni- & $\begin{array}{c}\text { Largo } \\
\mathbf{d e}\end{array}$ & Índice de & Doble & $\begin{array}{c}\text { Índice } \\
\text { de }\end{array}$ \\
\hline F.V & $\begin{array}{c}\text { Rendi- } \\
\text { miento }\end{array}$ & cloro & residual & ficación & rotura & Reventam. & plegado & rasgado \\
\hline & $\mathbf{( \% )}$ & $\mathbf{( \% )}$ & $\mathbf{( \% )}$ & $\mathbf{( \% )}$ & $\mathbf{( m )}$ & $\mathbf{( k P a * m 2 / g )}$ & $\begin{array}{c}\mathbf{1} \mathbf{~ k ~ d e} \\
\text { tensión) }\end{array}$ & $\mathbf{( m 2 / g )}$ \\
\hline E. viminalis & $70.9 \mathrm{a}$ & $25.6 \mathrm{~b}$ & $20.1 \mathrm{~b}$ & $23.6 \mathrm{~b}$ & $5278.3 \mathrm{c}$ & $67.7 \mathrm{a}$ & $15.7 \mathrm{c}$ & $27.6 \mathrm{c}$ \\
\hline E. glóbulus & $67.5 \mathbf{b}$ & $21.9 \mathbf{c}$ & $16.4 \mathbf{c}$ & $25.9 \mathbf{a}$ & $6384.3 \mathbf{a}$ & $75.6 \mathbf{a}$ & $48.5 \mathbf{~ a}$ & $35.9 \mathbf{a}$ \\
\hline $\begin{array}{r}\text { E. } \\
\text { amaldulensis }\end{array}$ & $65.5 \mathrm{c}$ & $26.35 \mathrm{a}$ & $22.2 \mathrm{a}$ & $21.3 \mathrm{c}$ & $5883 \mathrm{~b}$ & $68.5 \mathrm{a}$ & $35.6 \mathrm{~b}$ & $32.9 \mathrm{~b}$ \\
\hline
\end{tabular}

Para rendimiento se observa que E. viminalis tabla 2, obtuvo el mayor \% en relaciona a las demás especies esto se debe probablemente a las características anatómicas de la especies y no sufrió mayor degradación de los componentes químicos, que coincide con GOITIA (2005) Y KIRT (2001).Realizando Comparación con $E$. viminalis y E. camaldulensis, la degradación de la lignina y otros componentes de la madera de $E$. glóbulus, es menor en 5.45 y mayor en $3.38 \%$, con respecto a E. camaldulensis. De los resultado se indica que los rendimientos se encuentra se mantienen dentro de los límites considerados y aptos para producir pulpa para papel de diversos usos.
El consumo de reactivos químicos (índice de cloro), para blanquear pulpa de E. glóbulus, es menor en $3.7 \%$ con respecto a la pulpa de $E$. viminalis y menor en $4.45 \%$ con respecto a $E$. camaldulensi. Resultado que concuerda con los obtenidos por Trujillo (1983) y Bueno (2001). Así mismo se observa en pulpa de E. globulus menor porcentaje de lignina residual $(16.4 \mathrm{c}) \mathrm{y}$ mayor deslignificación (25.9 a), por lo que el consumo de reactivo (cloro), es menor para blanquear pulpa, es decir a menor porcentaje de lignina residual en pulpa el consumo de reactivos es menor para el blanqueo de la pulpa.

Los ensayos ajustados a $45^{\circ} \mathrm{SR}$ (choper Riegler), para longitud de rotura de pulpa semiquímica de E. glóbulus es mayor en 1.106 metros que la pulpa de $E$. viminalis y con respecto a $E$. camaldulensis en mayor en $501 \mathrm{~m}$, tabla 2, referente a índice de reventamiento doble plegado y índice de rasgado, la pulpa de $E$. glóbulus se mantiene con mayores resultados, dentro del ordenamiento de Duncan (a), que concuerda con los obtenidos por FAIREST (2004), PIZARRO (2004) y Bueno (2001). Que el refinado influye directamente en las propiedades físico-mecánicas del papel; manifiesta incrementando la resistencia a la tensión, dobles pliegues, estallido, transparencia y lisura del papel.

\section{CONCLUSIONES.}

Las características químicas (índice de cloro, deslignificación y lignina residual) en pulpa semiquímica de E. viminalis, E. glóbulus y E. camaldulensis, se encuentra dentro de los límites establecidos. El rendimiento en pulpa semiquímica al sulfato, varia de $70.90-65.40 \%$ en las tres especies en estudio, la pulpa de $E$. glóbulus alcanzó el mayor deslignificación, la pulpa de E. glóbulus, alcanzó los valores más altos, en propiedades de resistencia mecánica, largo de rotura, índice de reventamiento, dobles pliegues, aumentan significativamente por efecto del refinado en las tres especies 
estudiadas, haciendo adaptables para la producción de pulpa para papel, para diversos usos.

\section{AGRADECIMEINTO.}

Los autores agradecen al Dr. ENRIQUE GONZALES MORA, Jefe del Laboratorio de Pulpa y Papel de la Universidad Nacional Agraria La Molina. Por su apoyo y orientación para la culminación de este trabajo investigativo.

\section{REFERENCIAS BIBLIOGRÁFICAS.}

BUENO, JORGE. (2001). Estudio de posibilidades industriales de maderas nacionales para fabricación de pulpa para papel. Depto. de industrias Forestales. UNA-Ministerio de Industria y Turismo. Universidad Nacional Agraria La Molina. 98 p.

CARRUÑO, JOSE, y VILLEGAS, JORGE. (2003). Investigación sobre el proceso Sulfito Neutro para obtención de pulpa a partir de E. glóbulos. Instituto Forestal Latinoamericano de Investigaciones y Capacitación. Mérida. Venezuela p. 1245.

COSTA, TRUjILlo. (1983). Manual de fabricación de papel. Editorial. Bosch. Barcelona. Universidad de Barcelona. 89 $\mathrm{p}$.

DE LOS HEROS, M. (2003). Aptitud papelera de eucalipto de cuatro procedencias. Tesis para optar el Título de Ingeniero Forestal. UNA la Molina. Lima. Universidad Nacional Agraria La molina -Perú. $73 \mathrm{p}$.

FAIREST, R. (2004). Pulpa para papel a partir de tres maderas típicas de la selva del Estado de Bolívar. Año VII ( $\left.\mathrm{N}^{\circ} 10\right)$. Revista Forestal de Venezuela. Universidad de Mérida,p 121-141.
H I N O S T R OZA, D ONATO. (2001). Contribución al estudio de tres especies frondosas tropicales para producción de pulpa y papel, proceso al sulfato y sulfito neutro. Tesis para optar el título de Ingeniero Forestal. Universidad Nacional del Centro del Perú-Huancayo. $95 \mathrm{p}$.

GOITIA, LUIS. (2005). Evaluación de pulpa al sulfato de cinco especies forestales del Perú. Tesis para optar el Título de Ingeniero Forestal. Universidad Nacional Agraria. p 3-36.

GRANT, C. (2001). Manual sobre fabricación de pulpa y papel. Editorial Continental. México,p 46-125.

KIRT, ROBERT. (2001). Tecnología Química. Bases orgánicas e inorgánicas, Editorial Max Well. New York, p 65 - 750.

LIBBY, C. (2000). Ciencia y tecnología sobre pulpa y papel. Editorial Continental. México. Tomo I. 99 - 132. University of Chicago.

MOLINA, ENESTO. (2002). Pulpa al sulfato de siete mezclas de 39 especies forestales de la zona de Iquitos. Tesis para optar el Título de Ingeniero Forestal. Universidad Nacional Agraria. La Molina, p 12 - 27.

NAVARRO, J. (2006). Temas de fabricación del papel. Editorial Marfil. España. Universidad de Mérida, p 334-458.

PIZARDO, A. (2004). Influencia de tres variables en la obtención de pulpa química al sulfato de tangarana de altura (Sclerolobium paniculata). Tesis para optar el Título de Ingeniero Forestal. Universidad Nacional Agraria la Molina, 16 - 33.

RODRIGUEZ, J. (2001). Controles de fabricación de papel. Editorial Blume. Madrid, p 12-33.

TORRES, P. (2001). Influencia de sulfidez en pulpa semi-química al sulfato. Tesis para optar el Título de Ingeniero Forestal. Universidad Nacional Agraria, la Molina. 78 p. 
TRUJILLO, C. (2001). Pulpa química al sulfato de E. glóbulos Labill, de tres procedencias. Cajamarca, Cuzco y Junín. Tesis para optar el Título de Ingeniero Forestal. Universidad Nacional Agraria, La Molina, p 12-33.

\section{CORRESPONDENCIA}

Segundo Alipio Cruz Hoyos

Horacio Zevallos $\mathrm{N}^{\circ} 600$ - Jaén - Cajamarca Perú.

scruzhjerusalen@hotmail.com 